\title{
V2: Selbstberichtete Beschwerden von Pflegekräften in einem Krankenhaus der Schwerpunktversorgung
}

\author{
Kathleen Hirsch • Sebastian Lindenberg
}

Online publiziert: 29. Oktober 2013

(C) Springer-Verlag Wien 2013

Unzufriedenheit, Überlastung, Schlafprobleme, Beschwerden im Bereich des Bewegungsapparates oder psychische Erkrankungen aufgrund der Belastungen, die dieser Beruf mit sich bringt, sind unter Pflegekräften weit verbreitete Probleme. Doch nur Mitarbeiter, die zufrieden und motiviert ihrer Arbeit nachgehen, können dazu beitragen, dass Krankenhäuser unter den Anbietern von Gesundheitsleistungen dauerhaft bestehen können.

Aus diesem Grund wurde an einem Krankenhaus in Sachsen-Anhalt eine Befragung der Mitarbeiter durchgeführt. Auf Basis der Ergebnisse soll im Rahmen der Implementierung eines BGM in Erfahrung gebracht werden, welche Möglichkeiten bestehen, vorhandene Beschwerden zu lindern bzw. welche gesundheitsfördernden Maßnahmen in Zukunft angeboten werden können, um die Situation der Mitarbeiter langfristig zu verbessern.

Bei dem verwendeten Fragebogen handelte es sich um ein valides Erhebungsinstrument. Dabei wurden unter anderem demografische Daten wie Alter, Betriebszugehörigkeit, Belastungen am Arbeitsplatz, Zufriedenheit am Arbeitsplatz und Beschwerden im vergangenen Jahr erhoben. Die Rücklaufquote unter den Pflegekräften betrug 55,6\% (bei $n=168$ von $n_{\text {ges }}=302$ ). Die Befragung wurde vom 1.6.2011 bis 30.9.2011 durchgeführt. Die Auswertung erfolgte mit SPSS.
37,8\% $(n=62)$ der befragten Pflegekräfte gaben an, selten und 47,0\% (n=77) häufig unter Rückenschmerzen zu leiden. Unter dauerhaften Rückenschmerzen leiden 7,9\% ( $n=13$ ). 90,8\% aller Befragten klagen über Nackenbzw. Schulterschmerzen. Arbeiten in gebückter Haltung oder monotone Tätigkeiten wurde von 26,1\% $(n=44)$ bzw.11,9\% $(n=20)$ als erheblich bzw. stark belastend empfunden. Für die allgemeine Schlafqualität konnte ein Mittelwert von 6,45 ermittelt werden (Skala 0 für sehr schlechten und 10 für sehr guten Schlaf). 37,7\% $(n=92)$ der Pflegekräfte schlafen unruhig, mit Wachphasen. Nur 9,8\% $(n=24)$ gaben an, dass ihr Schlaf erholsam ist. 52 (31,9\%) und 6 $(3,7 \%)$ der befragten Pflegekräfte leiden häufig bzw. immer unter Kopfschmerzen.

Es konnten Korrelationen zwischen dem Arbeiten in gebückter Haltung bzw. dem Verrichten monotoner Tätigkeiten und Rückenbeschwerden festgestellt werden.

Auf Basis dieser Erhebung wurden gesundheitsfördernde Programme (sportliche und psychologische Angebote) und der Ausbau des Präventionsprogramms zur Vorbeugung von Rückenbeschwerden implementiert. Es erfolgen weiterführende Evaluationen zur Wirksamkeitsprüfung.
K. Hirsch $(\bowtie)$

Krankenpflegeschule des Harzklinikums D.-C.-Erxleben GmbH,

Quedlinburg, Deutschland

E-Mail: k.hirsch@klinikum-quedlinburg.de

S. Lindenberg

Hochschule Magdeburg-Stendal, Stendal, Deutschland 